\title{
Hospitalised hip fracture risk with rosiglitazone and pioglitazone use compared with other glucose-lowering drugs
}

\author{
H. M. Colhoun - S. J. Livingstone $\cdot$ H. C. Looker • \\ A. D. Morris - S. H. Wild • R. S. Lindsay $\cdot$ C. Reed • \\ P. T. Donnan • B. Guthrie - G. P. Leese - J. McKnight • \\ D. W. M. Pearson - E. Pearson - J. R. Petrie - S. Philip • \\ N. Sattar - F. M. Sullivan - P. McKeigue • \\ on behalf of the Scottish Diabetes Research Network \\ Epidemiology Group
}

Received: 16 March 2012 / Accepted: 25 June 2012 /Published online: 4 September 2012

(C) The Author(s) 2012. This article is published with open access at Springerlink.com

\begin{abstract}
Aims/hypothesis Current drug labels for thiazolidinediones (TZDs) warn of increased fractures, predominantly for distal fractures in women. We examined whether exposure to TZDs affects hip fracture in women and men and compared the risk to that found with other drugs used in diabetes. Methods Using a nationwide database of prescriptions, hospital admissions and deaths in those with type 2 diabetes in Scotland we calculated TZD exposure among 206,672 individuals. Discrete-time failure analysis was used to model the
\end{abstract}

Members of the SDRN Epidemiology Group beyond the authors listed: I. Brady, Medical Research Institute, University of Dundee;

J. Chalmers, Victoria Hospital, Kirkcaldy; S. Cunningham, Clinical

Technology Centre, University of Dundee; R. Elder, Information

Services Division, NHS Scotland; A. Emslie-Smith, Arthurstone

Medical Centre, Dundee; L. Govan, Institute of Health and Wellbeing, University of Glasgow; D. Levin, Medical Research Institute, University of Dundee; R. McAlpine, Diabetes Managed Clinical Network, NHS Tayside

H. M. Colhoun $(\bowtie) \cdot$ S. J. Livingstone $\cdot$ H. C. Looker $\cdot$

A. D. Morris • P. T. Donnan • B. Guthrie • G. P. Leese $•$

E. Pearson $\cdot$ F. M. Sullivan

Medical Research Institute, University of Dundee,

Ninewells Hospital \& Medical School,

Dundee, Scotland DD1 9SY, UK

e-mail: h.Colhoun@dundee.ac.uk

H. M. Colhoun

NHS Fife, Hayfield House,

Kirkcaldy, Fife, UK

S. H. Wild $\cdot$ C. Reed $\cdot$ J. McKnight $\cdot$ P. McKeigue

Centre for Population Health Sciences, University of Edinburgh,

Edinburgh, UK effect of cumulative drug exposure on hip fracture during 1999-2008.

Results There were 176 hip fractures among 37,479 exposed individuals. Hip fracture risk increased with cumulative exposure to TZD: OR per year of exposure 1.18 (95\% CI $1.09,1.28 ; p=3 \times 10^{-5}$ ), adjusted for age, sex and calendar month. Hip fracture increased with cumulative exposure in both men (OR 1.20; 95\% CI 1.03, 1.41) and women (OR 1.18 ; $95 \%$ CI $1.07,1.29)$ and risks were similar for pioglitazone (OR 1.18) and rosiglitazone (OR 1.16). The

R. S. Lindsay $\cdot$ J. R. Petrie $\cdot$ N. Sattar

University of Glasgow,

Glasgow, UK

J. McKnight

NHS Lothian, Western General Hospital,

Edinburgh, UK

D. W. M. Pearson - S. Philip

Grampian Diabetes Research Unit, Woolmanhill Hospital, Aberdeen, UK 
association was similar when adjusted for exposure to other drugs for diabetes and for other potential confounders. There was no association of hip fracture with cumulative exposure to sulfonylureas, metformin or insulin in this analysis. The 90-day mortality associated with hip fractures was similar in ever-users of TZD (15\%) and in never-users (13\%). Conclusions/interpretation Hip fracture is a severe adverse effect with TZDs, affecting both sexes; labels should be changed to warn of this. The excess mortality is at least as much as expected from the reported association of pioglitazone with bladder cancer.

Keywords Fractures · Hip fracture · Pharmacoepidemiology · Thiazolidinedione $\cdot$ Type 2 diabetes

$\begin{array}{ll}\text { Abbreviations } \\ \text { EMA } & \text { European Medicines Agency } \\ \text { FDA } & \text { Food and Drugs Administration } \\ \text { NHS } & \text { National Health Service } \\ \text { NRS } & \text { NHS Research Scotland } \\ \text { SCI-DC } & \text { Scottish Care Information-Diabetes Collaboration } \\ \text { TZD } & \text { Thiazolidinedione }\end{array}$

\section{Introduction}

Based on its association with cardiovascular disease, the thiazolidinedione (TZD) rosiglitazone has been withdrawn in Europe and is on restricted licence in the USA. Last year the prescribing information for pioglitazone was updated by the European Medicines Agency (EMA) and other regulatory agencies because of reports of a risk ratio for bladder cancer ranging from 1.12 to 1.33 after 2 years' exposure. Pioglitazone was stated to be a valid treatment option for certain patients with type 2 diabetes, specifically when metformin has not been suitable or has failed to work adequately [1-3].

However, another important adverse effect of TZD therapy is bone fracture. That TZDs can cause fracture was first suggested by the ADOPT clinical trial, in which rosiglitazone treatment increased distal fracture risk in women only [4]. Since then other trials [5-7] and observational studies [8-13] have reported on fracture risk with TZD. These studies are generally consistent in finding an increased risk in older women for distal fracture but they are inconsistent with respect to effects in men and effects on hip fractures. Establishing whether TZDs cause hip fracture is important given the high rates of mortality associated with hip as compared with distal fractures [14, 15]. Few studies have had adequate power to examine these effects and none have adjusted for prior exposure to all other main classes of drugs for diabetes. Meanwhile, the drug labels continue to emphasise that TZD-associated fracture risk is predominantly for distal fractures in women. In this analysis we have used data from a Scotland-wide diabetes register to examine whether cumulative exposure to TZDs also affects hip fracture rates in women and in men, whether exposure to other glucoselowering drugs explains any associated risks and to compare the risks associated with rosiglitazone and pioglitazone.

\section{Methods}

Ethical approval Approval was obtained from the Scotland A Research Ethics Committee, the Caldicott (data privacy) Guardians for the 14 Scottish Health Boards and the NHS Information Services Division Privacy Advisory Committee.

Data sources In Scotland patients with type 2 diabetes are registered on a nationwide clinical record system that also includes issued prescriptions and other clinical data from primary care and diabetes clinics-the Scottish Care Information-Diabetes Collaboration (SCI-DC) database [16]. SCI-DC data are linked anonymously to hospital admissions data (Scottish Morbidity Record SMR-01), held by the Information Services Division of the National Health Service (NHS), and death data held by the General Register Office for Scotland, using the healthcare number with probabilistic linkage (mal-linkage rate $<3 \%$ ) [17]. From the linked data, we extracted data on hip fractures. We used the standard ICD-10 codes (www.who.int/classifications/ icd/en/) used by the Information Services Division of the NHS and we excluded admissions containing codes for traffic accidents and other severe external causes that might be expected to cause fractures even in individuals not susceptible to fracture (see Appendix for ICD-10 codes used).

Cohort under study and dates of entry and exit from followup We extracted anonymised data on all drug prescriptions and potential confounders from SCI-DC for all patients with type 2 diabetes ever registered on SCI-DC who were under observation for prescribing records in SCI-DC at some time during 1999-2008 $(n=225,821)$. Using the prescription data we defined all stretches of exposure to TZD, metformin, insulin and sulfonylurea for each patient. We defined the period of follow-up for the analysis as being from 1 January 1999 (the year in which TZD prescribing started in Scotland) to 2008 (the most recent data available to us); thus all TZD users included in the analysis are incident users. To model the univariate effects of TZD, we selected all individuals who were evaluable for cumulative exposure to TZDs (i.e. anyone with more than 1 year of diabetes in which drug exposure was unknown since introduction of TZDs in 1999 was excluded) giving a total cohort of 206,672 individuals. To model the joint effects of all main 
classes of glucose-lowering drugs (sulfonylureas, metformin, insulin, TZD), the analysis was restricted to a subset of 173,113 individuals who first came under observation for drug exposures no more than 1 year after diagnosis of diabetes (i.e. who were evaluable for cumulative exposure to all classes of drugs for diabetes). For each individual the date of entry into the analysis was the latest of three dates: first date under observation for drug prescribing, 1 January 1999 or date of diabetes diagnosis. The exit date was the earliest of four dates: date of first hip fracture, date of death, last date under observation or 31 December 2008.

Data preparation for modelling and calculation of exposure The period of follow-up (1 January 1999 to 31 December 2008) was divided into discrete intervals of 28 days and occurrence of hip fracture in any interval was scored as a binary variable. A data matrix was generated with one row for each individual under observation in each 28-day interval, and columns specifying event status (coded as binary), fixed covariates (sex, prior fracture at any site) and timevarying covariates (age, calendar month, ever exposed to drug of interest by the start of the interval and cumulative exposure to each drug). For each drug class, cumulative exposure at the start of each interval was calculated as a sum over all earlier intervals in which a drug prescription was current. The exposure duration was calculated from the first and last dates of a run of repeat prescriptions at a given dose plus the duration of the last prescription in that run. Gaps between runs of repeat prescriptions did not accumulate exposure. Following drug cessation the cumulative days of exposure at cessation was then carried forward in subsequent time intervals. Gaps in being under observation for prescribing records (possibly representing temporary absence from Scotland) were ignored when defining the first and last dates under observation, as such gaps represented less than $5 \%$ of all person-years between first and last prescription records.

Statistical methods The effects of cumulative exposure were evaluated in a discrete-time survival model $[18,19]$; this is set up as a logistic regression model in which each individual contributes one observation for each 28-day interval between their entry and exit dates. We must use discrete time intervals in any case to model time-varying covariates such as cumulative exposure, and with short intervals of equal length very little information is lost by encoding the failures within each interval as binary (for logistic regression) rather than as times to failure within each interval (for Poisson regression). The model included age, sex, calendar month, ever-exposure to one or more drugs under study and cumulative exposure to the same drugs. This model was then run for each sex separately and with separate terms for pioglitazone and rosiglitazone. To evaluate the effect of dose we compared the deviance of the basic model in which the cumulative exposure was calculated as years of exposure (ignoring dose) to a model in which the cumulative exposure was calculated as the sum of daily doses. We evaluated the effects of other diabetes drugs and covariates by including terms for ever-exposure and time-dependent cumulative exposure to sulfonylureas, metformin and insulin, timedependent terms for time since diagnosis of diabetes, and for baseline postmenopausal oestrogen therapy, prior cardiovascular disease, prior fracture at any site, and exposure to thyroid drugs, loop diuretics, steroids, tricyclic antidepressants and bisphosphonates. These potential confounders were chosen based on the QFRACTURE fracture prediction model [20], data availability and prior information about drug effects on bone metabolism [21]. Smoking status before entry was recorded for just $61 \%$ of individuals under study and $\mathrm{BMI}$ and $\mathrm{HbA}_{1 \mathrm{c}}$ levels before entry were available for less than one-quarter of individuals.

Evaluation of drug effect Comparisons of fracture risk between ever-users and never-users of a drug are likely to be confounded by 'frailty', in that those prescribed and those not prescribed the drug may differ in their prior susceptibility to fracture. Such 'allocation bias' or 'confounding by indication' cannot reliably be dealt with by simple adjustment for measured covariates such as past medical history. One way to be certain that an estimate of a drug's causal effect cannot be due to such between-person confounding is to model the data with two terms in the model: one a timeupdated term for ever-exposure up to that point in time and one for time-updated cumulative exposure. The everexposed term contrasts the rate in unexposed person timeperiods with the predicted rate in person time-periods when exposure has just started. It is the sum of any stepwise (immediate) effect of drug exposure on fracture and the confounding effect of time-invariant frailty (i.e. allocation bias). It can be shown algebraically that with the ever-use term in the model the likelihood of the cumulative effect term does not depend on the events in the unexposed and therefore cannot be affected by allocation bias. Thus we focus the inference of causality on the cumulative term. If the drug does in fact cause an immediate increase in fracture risk, then our conclusions based only on the cumulative term are conservative.

\section{Results}

Comparison of exposed and unexposed individuals of the 206,672 individuals whose cumulative TZD exposure could be evaluated, 37,479 had accumulated some exposure to TZDs by the end of follow-up. Table 1 shows comparison of characteristics at entry to the cohort between those never- 
Table 1 Comparison of characteristics at time of entry to the study between those never-users and those eventual users of TZDs $(n=206,672)$

\begin{tabular}{|c|c|c|c|}
\hline Characteristic & $\begin{array}{l}\text { Never-users of TZD } \\
(n=169,193)\end{array}$ & $\begin{array}{l}\text { Ever-TZD users } \\
(n=37,479)\end{array}$ & $\begin{array}{l}p \text { value for age-adjusted } \\
\text { difference }^{\mathrm{a}}\end{array}$ \\
\hline Age (years), median (interquartile range) & $65.2(57.5-73.3)$ & $58.3(57.5-65.5)$ & $<0.001$ \\
\hline $\begin{array}{l}\text { No. of years since diagnosis of diabetes, median } \\
\text { (interquartile range) }\end{array}$ & $0(0-2.25)$ & $0.1(0-4.0)$ & $<0.001$ \\
\hline Female, \% (no.) & $47(78,977)$ & $46(17,374)$ & $<0.001$ \\
\hline \multicolumn{4}{|c|}{ Current/past use of glucose-lowering drugs at entry to cohort, \% (no.) } \\
\hline TZD & 0 by definition & $0.2(65)$ & \\
\hline Metformin & $10(16,923)$ & $17(6,546)$ & $<0.001$ \\
\hline Sulfonylureas & $14(24,303)$ & $21(7,910)$ & $<0.001$ \\
\hline Insulin & $4(6,014)$ & $1(306)$ & $<0.001$ \\
\hline Prior admission for fracture at any site, $\%$ (no.) & $5(8,706)$ & $4(1,612)$ & $<0.001$ \\
\hline Prior admission for cardiovascular disease, \% (no.) & $17(28,837)$ & $12(4,477)$ & $<0.001$ \\
\hline \multicolumn{4}{|l|}{ Current/past use of other drugs, \% (no.) } \\
\hline Steroids & $8(13,983)$ & $8(2,978)$ & 0.6 \\
\hline Thyroid drugs & $4(7,186)$ & $4(1,468)$ & $<0.001^{\mathrm{b}}$ \\
\hline Tricyclic antidepressants & $7(12,077)$ & $7(2,565)$ & 0.18 \\
\hline Postmenopausal oestrogen (women only) & $8(5,995 / 78,977)$ & $12(2,113 / 17,374)$ & $<0.001$ \\
\hline Bisphosphonates & $1(1,146)$ & $0.3 \%(128)$ & 0.005 \\
\hline Loop diuretics & $14(23,524)$ & $10(3,824)$ & 0.33 \\
\hline Current smoking & $30(31,163 / 103,271)$ & $30(7,067 / 23,735)$ & $<0.001^{\mathrm{b}}$ \\
\hline
\end{tabular}

${ }^{\mathrm{a}} p$ values are for the age-adjusted difference other than for age itself

${ }^{\mathrm{b}}$ Although the crude rates for thyroid drug use and smoking are the same, the smoking rate in ever-users of TZDs is lower and use of thyroid drugs is higher than expected given their younger age (i.e. adjusted for age, smoking is significantly lower and thyroid drug use is significantly higher)

users and those eventual users of TZDs during the period of follow-up, with $p$ values, other than for the age comparison, based on adjustment for age. Ever-users of TZD were significantly younger than never-users. Adjusted for age, in ever-users vs never-users of TZDs the proportion with prior admission coded with cardiovascular disease was lower, the proportion who had used bisphosphonates was lower and the proportion of women who had used postmenopausal oestrogens was higher, even after adjusting for the difference in age at entry. Although the crude proportion of females among the TZD users was similar to never-users, after adjustment for the age difference in users there was a higher proportion of females among TZD users than would be expected. Although crude smoking rates and thyroid drug usage rates are the same in the two groups, adjusted for the younger age of users, smoking is less common and thyroid drug use more common than would be expected in TZD users compared with never-users.

Effects of cumulative exposure There were 2,433 first hip fracture events: 98 in current users of a TZD at the time of fracture, 78 in ex-users and 2,258 in never-users. Of the 176 hip fractures in users, 44 were in men and 132 in women. Figures 1 and 2 show the rates of hip fracture admission in women and men by cumulative exposure to TZD standardised (within 10-year age bands) to the age distributions (over all person-years) of all women and all men under study, respectively. In both women and men the fracture rate in the first year of exposure is lower than the overall fracture rate in the neverexposed periods, consistent with the comparisons in Table 1, which suggest that those eventual users of TZDs are likely to be in better health (and thus presumably at less prior risk of fracture) than those never exposed to TZDs. The plots show that fracture rate rises with increasing exposure; while the data fit a linear model and we use this linear term to describe the effect per cumulative year of exposure subsequently, the graphs also suggest a lag before fracture risk starts to increase, and a flattening of the curve after 4 years of exposure. Consistent with this a post hoc comparison, comparing rates with 2-4 years and $>4$ years exposure with 1 day -2 years exposure, gave estimates of OR 1.87 (95\% CI 1.35, 2.61) and 1.94 (95\% CI 1.28, 2.94), respectively, overall, with ORs of $1.72(95 \%$ CI $1.17,2.52)$ and 1.85 (95\% CI $1.14,3.00)$ in women and ORs of $2.44(95 \%$ CI $1.27,4.7)$ and $2.28(95 \%$ CI $0.99,5.25)$ in men for these categories of exposure.

The terms for ever-exposure and cumulative exposure in the discrete-time failure model can be given a visual interpretation in this plot, which superimposes the regression line for the linear effect of cumulative exposure $(x)$ (see Fig. 1 


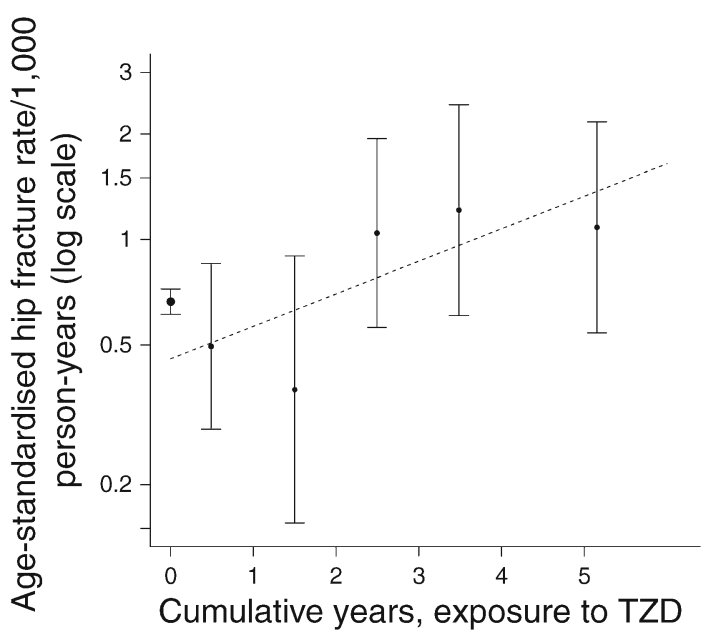

Fig. 1 Age-standardised rates of hip fracture by cumulative exposure to TZD in women. The error bars indicate the $95 \% \mathrm{CI}$ for the rates. The $x$ axis shows cumulative years of exposure; the data point at $x=0$ is for all unexposed person time-periods, and the other data points are for exposure categories $0<x \leq 1,1<x \leq 2,2<x \leq 3,3<x \leq 4$ and $x>4$ years. The dotted regression line shows the linear effect of cumulative exposure $(x)$ calculated by weighted least squares from the ever-exposed data points as an approximation to the modelling approach described in the Methods. Whereas the data point at $x=0$ is the log fracture rate observed for all unexposed person time-periods, the point on the dotted regression line where $x=0$ is the estimate from the model of the log fracture rate at the point of starting exposure in those exposed. Thus the difference in height between these two points gives the magnitude of the ever-exposed term and is the sum of any immediate stepwise effect of the drug and any difference in prior risk of fracture in ever vs never exposed. Since an immediate stepwise effect of TZD on hip fracture is unlikely the difference in height suggests that those who become exposed have a lower prior fracture risk than the never exposed

legend). Since an immediate stepwise effect of TZDs on hip fracture is unlikely, the difference in height between the regression line at $x=0$ and the observed data point at $x=0$

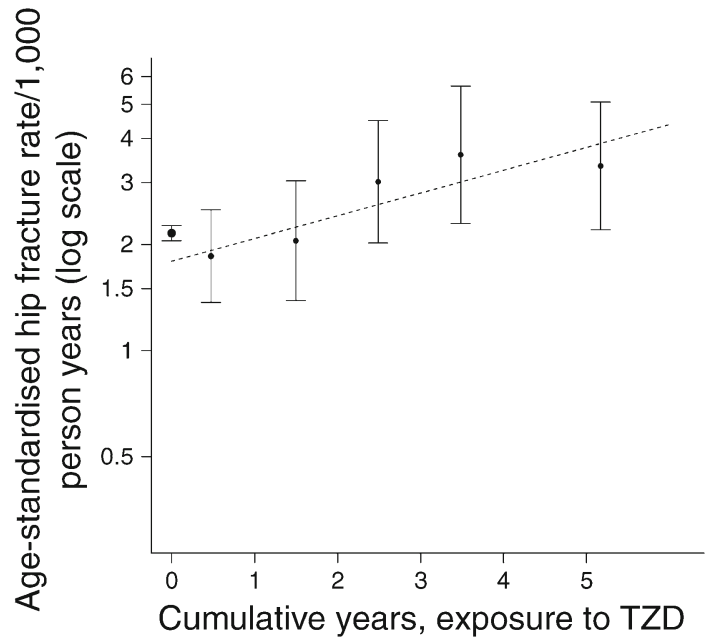

Fig. 2 Age-standardised rates of hip fractures by cumulative exposure to TZD in men. The error bars indicate the $95 \%$ CI for the rates. See legend to Fig. 1 for interpretation of this figure suggests that those who become exposed have a lower prior fracture risk than those never exposed.

The OR for hip fracture with each cumulative year of TZD exposure was $1.18\left(95 \%\right.$ CI $\left.1.09,1.28 ; p=3 \times 10^{-5}\right)$ in both sexes combined, adjusted for age, sex, calendar month and ever-use of TZDs. The effect of cumulative exposure to TZDs on hip fracture did not differ between men (OR 1.20 per year exposure, $95 \%$ CI 1.03, 1.41, based on 44 events in exposed men) and women (OR 1.18, 95\% CI 1.07, 1.29, based on 132 events in exposed women). In a joint analysis with cumulative and ever-use terms for both TZDs, the effect per year of cumulative exposure did not differ between pioglitazone (OR 1.18; 95\% CI 1.00, 1.40) and rosiglitazone (OR 1.16; 95\% CI 1.06, 1.26). In comparison with the model in which cumulative exposure was coded as years of exposure, the deviance of the model in which cumulative exposure was coded as total cumulative dose (standardised as described in the Methods section) was three units higher, indicating that the model fit was better for exposure coded as cumulative years than cumulative milligram dose. In an analysis with follow-up censored on cessation of TZD the effect of cumulative exposure was slightly greater, consistent with a dropping off of effect on cessation (OR 1.33, 95\% 1.10, $1.61, p=0.003)$. Using a Cox regression rather than discrete time model gave the same estimate of effect for cumulative exposure as the main model (OR 1.18, $p=3 \times 10^{-5}$ ).

Over the entire period that individuals were under observation, the 90-day case fatality for hip fracture was similar at $15 \%$ in ever-users of TZDs and $13 \%$ in never-users.

Multivariate analysis including other drugs for diabetes Table 2 summarises the results of models of hip fracture risk in the subset of 173,113 individuals who were evaluable for cumulative exposure to all four glucose-lowering drug classes. In model 1 adjusted for sex, age, calendar month and ever-exposure, the OR per year of cumulative exposure to TZDs was 1.21 (95\% CI 1.09, 1.35). With additional adjustment for years since diagnosis, prior cardiovascular disease, prior admission for fracture, past exposure to drugs likely to affect fracture risk, and cumulative and ever-use terms for sulfonylureas, metformin and insulin (model 2), the OR for hip fracture per year of cumulative exposure to TZDs was 1.19 (95\% CI 1.07, 1.32). Hip fracture was positively associated with years since diagnosis of diabetes, prior admission for fracture, use of bisphosphonates and use of tricyclic antidepressants, and inversely associated with use of postmenopausal oestrogens in women. Further adjustment for statins, benzodiazepines, opiates, proton-pump inhibitors, thiazides, beta blockers, ACE inhibitors and angiotensin II (AII) receptor antagonists did not alter the conclusions (OR 1.15, $p=0.004$ ). In the $61 \%$ in whom smoking status data was available, on adjustment for smoking the effect remained significant (OR 1.12, $p=0.02$ ). 
Table 2 Discrete-time failure models for first hip fracture in cohort restricted to those first observed within 1 year of diagnosis of diabetes $(n=173,133)$

Model 1: association of hip fracture with cumulative years exposure to TZD with adjustment for sex and time-varying covariates (age, calendar month, ever-use of TZD). See Methods for the interpretation of the ever-use term

Model 2: Model 1 plus adjustment for additional baseline covariates (prior cardiovascular disease, prior fracture, past use of fracture-associated drugs) and additional time-varying covariates (years since diagnosis, ever-use and cumulative use of three other classes of glucoselowering drugs [sulfonylureas, metformin, insulin])

See Methods for the interpretation of the ever-use term

\begin{tabular}{|c|c|c|c|c|}
\hline Model & OR & CI, lower & CI, upper & $p$ value \\
\hline \multicolumn{5}{|l|}{ Model 1} \\
\hline Women vs men & 2.44 & 2.17 & 2.74 & $<0.001$ \\
\hline Age years & 1.12 & 1.11 & 1.12 & $<0.001$ \\
\hline Calendar years & 1.03 & 1.01 & 1.05 & 0.004 \\
\hline Ever TZD & 0.69 & 0.50 & 0.95 & 0.024 \\
\hline Cumulative TZD (effect per year) & 1.21 & 1.09 & 1.35 & $<0.001$ \\
\hline \multicolumn{5}{|l|}{ Model 2} \\
\hline Sex & 2.39 & 2.12 & 2.70 & $<0.001$ \\
\hline Age years & 1.11 & 1.10 & 1.12 & $<0.001$ \\
\hline Calendar years & 1.04 & 1.02 & 1.06 & $<0.001$ \\
\hline Years from diagnosis & 1.08 & 1.05 & 1.11 & $<0.001$ \\
\hline Prior fracture & 2.08 & 1.77 & 2.43 & $<0.001$ \\
\hline Prior cardiovascular disease & 1.02 & 0.90 & 1.17 & 0.710 \\
\hline \multicolumn{5}{|l|}{ Past drug exposure } \\
\hline Steroid & 0.95 & 0.80 & 1.12 & 0.55 \\
\hline Post-menopausal oestrogen & 0.60 & 0.42 & 0.86 & 0.005 \\
\hline Tricyclic & 1.47 & 1.25 & 1.72 & $<0.001$ \\
\hline Thyroid drugs & 0.95 & 0.78 & 1.15 & 0.59 \\
\hline Bisphosphonates & 1.69 & 1.22 & 2.33 & 0.001 \\
\hline Loop diuretics & 1.07 & 0.95 & 1.21 & 0.25 \\
\hline \multicolumn{5}{|l|}{ Diabetes drug exposure } \\
\hline Ever sulfonylurea & 1.31 & 1.14 & 1.52 & $<0.001$ \\
\hline Ever metformin & 0.75 & 0.64 & 0.87 & $<0.001$ \\
\hline Ever insulin & 1.13 & 0.83 & 1.55 & 0.43 \\
\hline Ever TZD & 0.61 & 0.44 & 0.85 & 0.003 \\
\hline Cumulative sulfonylurea years & 1.01 & 0.99 & 1.04 & 0.440 \\
\hline Cumulative metformin years & 1.02 & 1.00 & 1.05 & 0.095 \\
\hline Cumulative insulin years & 1.03 & 0.96 & 1.11 & 0.35 \\
\hline Cumulative TZD years & 1.19 & 1.07 & 1.32 & 0.001 \\
\hline
\end{tabular}

The model shows that there is no significant association of cumulative exposure to other glucose-lowering drugs with hip fracture. The ever-use terms for other drugs are consistent with allocation to sulfonylureas and insulin being associated with greater fracture susceptibility and allocation to metformin being associated with lower fracture susceptibility.

\section{Discussion}

In this nationwide cohort TZD treatment was associated with an $18 \%$ relative increase in hip fracture rates for every cumulative year of TZD exposure, in both men and women. The risk was similar for rosiglitazone and pioglitazone. There was no evidence that cumulative exposure to insulin, sulfonylurea or metformin were independently associated with hip fracture.
Our data add to growing evidence from observational studies and trials on TZD-associated fracture and serve to clarify several remaining questions about this risk [4-13, 22]. Our study was sufficiently large to be able to demonstrate effects in men and on hip fracture, for both of which smaller studies in the literature have reported inconsistent findings. Our study included more male TZD users $(20,105$ vs 3,064$)$ than the extensive meta-analysis of randomised trial data carried out by Loke et al [13] and this is likely to explain why we have detected effects in men not apparent in trials. A previous cohort study found that total fractures were increased in men but included few patients over 65 years old and so was underpowered for detecting effects on hip fracture [8]. One self-controlled case series study reported an increase in hip fracture in women and total fracture in men though the effect on hip fracture in men did not reach statistical significance [22]. The self-controlled case series design minimises between subject allocation bias but the method as originally 
conceived assumes that the observation time for each case is independent of the event time. Where the event has a high mortality rate (as we have shown is the case with hip fracture) this assumption is violated. Recent modifications to the method render it less sensitive to assumption but the modified method remains sensitive to the assumption that exposure is not event dependent-in the case of hip fracture and TZDs this assumption cannot be made; TZD exposure is less likely once fracture has occurred [23]. One case-control study with 18,000 fracture cases found effects on total and hip fracture in women and no overall or hip effect in men but the levels of TZD exposure in men were low (only 1,971 exposed men were included) [24]. A recent report also found evidence of a cumulative effect of TZDs on total fracture but not hip fracture [25].

The data reported here also clarify that the fracture risks cannot be avoided by using pioglitazone instead of the nowrestricted rosiglitazone. Three previous studies reported similar hazard rates for both drugs [8], with one reporting larger effects for pioglitazone [9] and one larger effects for rosiglitazone [26]. However, none of them adjusted for exposure to the other TZD drug in their analysis as we did, so carry-over effects could not be excluded. In the previous study that involved an analysis for pioglitazone restricted to those with no prior exposure to rosiglitazone, pioglitazone was clearly associated with an increased risk [22]. Another strength of our study is that we have also simultaneously modelled the effects of cumulative exposure to TZDs, metformin, sulfonylureas and insulin, thus ensuring that the association of fractures with TZDs cannot be attributed to these other diabetes drugs. This is important because previous observational studies were unable to adjust for timevarying exposure to all these drugs. Thus our data add to existing data and provide strong evidence for causality of the TZD's effect because of its magnitude and because of no similar cumulative effect with other glucose-lowering drug classes being evident.

Inference of adverse drug effects from observational data presents methodological challenges, especially confounding of the association of drug allocation with outcome by susceptibility. In Scotland, TZD recipients have a more favourable profile of risk factors for fracture (less use of thyroid drugs, less apparent need for bisphosphonates, more use of postmenopausal oestrogens, fewer previous admissions for fracture) and appear to be in better health (fewer previous admissions for cardiovascular disease) than non-users at the same age. Also the estimates of the 'ever' term for TZD exposure from the model are consistent with those being allocated TZD having a lower fracture risk than non-users at the outset of TZD exposure (as they are for metformin too). Thus a simple comparison of ever vs never-users of TZDs would be confounded by indication (i.e. subject to allocation bias). For this reason we have based inference only on the cumulative effect of TZD on risk since this is not affected by confounders that are not time-varying. This assumes that drug exposure has no stepwise effect on fracture risk but, as bone mass changes only slowly, this is a reasonable assumption unless the drug causes falls, which is unlikely since TZDs do not cause acute hypoglycaemia. The post hoc analysis categorising cumulative exposure into 1 day to 2 years, $2-4$ years and $\geq 4$ years suggests that some threshold of cumulative exposure may be needed before effects are apparent. The greater effect when follow-up is censored at cessation is consistent with a dropping off of the effect when the drug is stopped, though we have not modelled this explicitly. Thus the effects at a given level of ongoing exposure may be higher than we estimate. Of course while we have shown a dose-response effect that seems specific to TZDs these are not the only criteria for causality. It remains possible that longterm glitazone users may differ from short-term users in their base risk of hip fractures. Relevant in assessing causality too is that there is a biological rationale; while there are several possible mechanisms through which TZDs might lead to fracture (e.g. by increasing falls) there is considerable evidence that peroxisome proliferator-activated receptor gamma (PPAR- $\gamma$ ) activation with TZDs leads to unbalanced bone remodelling with bone resorption increasing and bone formation decreasing [27].

A limitation of our analysis is that inference based on cumulative exposure does not exclude confounding by glycaemia. Although we do not have good coverage of $\mathrm{HbA}_{1 \mathrm{c}}$ in the first few years of follow-up, even if we had complete $\mathrm{HbA}_{1 \mathrm{c}}$ data simply adjusting for $\mathrm{HbA}_{1 \mathrm{c}}$ would be incorrect since it potentially determines exposure and is also altered by exposure [28]. A much better test of whether $\mathrm{HbA}_{1 \mathrm{c}}$ is likely to be a relevant confounder here therefore is the specificity of association; if $\mathrm{HbA}_{1 \mathrm{c}}$ were a confounder then we would expect to also see such confounding causing an apparent relationship with other glucose-lowering drugs and fractures risk, which is not the case.

It is vital to consider the importance of the elevated risk of hip fracture compared with the possible elevated risk of bladder cancer in users of pioglitazone, which has recently led to a label change by the US Food and Drugs Administration (FDA) and EMA. The FDA estimated that the risk difference for bladder cancer associated with exposure to pioglitazone for more than 12 months was 28 cases per 100,000 person-years of follow-up [29]. Five-year mortality for bladder cancer is about $50 \%$ [30]. For comparison, an approximate estimate of the absolute risk difference for hip fracture in TZD users can be obtained from Figs 1 and 2 by comparing the age-standardised rate at, say, $0-2$ years exposure with $2-4$ years exposure. This risk difference is approximately 1.4 per 1,000 person-years in women and 
0.7 per 1,000 person-years in men. Consistent with the established literature $[14,15]$, we found high 90-day casefatality rates of $15 \%$ in TZD users, similar to rates in neverusers. Based on this case-fatality information we can thus estimate that TZD use for 2 years or more will carry an excess mortality of at least 21 per 100,000 woman-years from hip fracture, compared with an estimated excess mortality of about 14 per 100,000 person-years from bladder cancer in those exposed to pioglitazone.

Thus, hip fracture should be considered at least as important a potential adverse effect of pioglitazone exposure as bladder cancer to be balanced against its efficacy in reducing $\mathrm{HbA}_{1 \mathrm{c}}$. Of note the recent EMA risk-benefit assessment of pioglitazone carried out following the emergence of bladder cancer data does not even mention fracture risk [31]. This suggests that the importance of this adverse effect is not fully appreciated and should be emphasised to clinicians and patients. Current TZD drug labels state that 'the risk of fracture should be considered' but emphasise that effects are on distal fracture and mostly in women. These labels should be changed to reflect the accumulated data on hip fracture and risk in men.

Acknowledgements We thank the diabetes patients in Scotland and the SCI-DC and NHS National Services Information Services Division Scotland who provided data for this study.

Funding This work was supported by the Wellcome Trust through the Scottish Health Informatics Programme (SHIP) Grant (Ref WT086113), the Chief Scientist Office Scotland and NHS Research Scotland (NRS). This study and its publication was carried out independently of the funders.

Duality of interest S. J. Livingstone, H. C. Looker, A. D. Morris, S. H. Wild, C. Reed, P. T. Donnan, B. Guthrie, G. P. Leese, D. W. M. Pearson, S. Philip, N. Sattar and F. M. Sullivan declare: no support from any organisation for the submitted work; no financial relationships with any organisations that might have an interest in the submitted work in the previous 3 years, no other relationships or activities that could appear to have influenced the submitted work. None of the authors received support from any organisation for the submitted work; however, the following authors have financial relationships with a variety of companies as listed below but do not consider any of these directly relevant to this work: H. M. Colhoun has received research support from Roche, Pfizer, Lilly, Boehringer Ingelheim and AstraZeneca as part of an EU Innovative Medicines Initiative research grant. She has also been part of the speaker's bureau and acted as a consultant for Pfizer, acts as a consultant for Lilly and owns Roche stocks and shares. None of these activities relate directly to this manuscript. R. S. Lindsay is an advisory board member for Lilly and Novo Nordisk and has received lecture fees from Novo Nordisk. J. McKnight is the Principal Investigator for a number of studies of new drugs, including drugs produced by companies that are likely to be in competition with glitazone therapy. These include Novo Nordisk, Lilly, Merck and Boehringer Ingelheim. However, this has not influenced his view or contribution to this manuscript. E. Pearson has received payment via his institution to support attendance at meetings and fees for speaking from Novo Nordisk, Lilly, Boehringer Ingelheim and BMS/AstraZeneca. He has also received grant funding to his institution from Merck
Serono. J. R. Petrie has received support for consultancy from Takeda, GSK, Novo Nordisk and sanofi-aventis. He also receives research support from Merck Serono and travel support from Takeda, GSK, Novo Nordisk and sanofi-aventis. P. McKeigue has received research support from the Wellcome Trust.

Contribution statement All authors made substantial contributions to the conception and design, acquisition of data or analysis and interpretation of data, as well as drafting or revising the manuscript. In detail, HMC specified the hypothesis and design of the study and drafted and edited the manuscript. SJL contributed to the data analysis and revision of the manuscript. HCL contributed to the interpretation of the data and revising the manuscript. SHW obtained funding and approvals for data linkage, contributed to analysis and interpretation of the data and revision of the manuscript. RSL chaired the collaborative group with oversight on data analysis, assisted with data collection and revised the manuscript. CR was involved in method development for the analysis and contributed revisions to the manuscript. PTD contributed to the design of the study and advised on the analysis and drafting of the article. BG contributed to the planning of the analysis and to the writing of the manuscript. GPL and JRP contributed to the interpretation of the data and revisions of the manuscript. JM, DWMP, EP and NS contributed to data acquisition, data interpretation and revisions to the manuscript. SP contributed to the data acquisition, study design and revisions to the manuscript. FMS contributed to the data processing, data interpretation and drafting of the manuscript. PM undertook primary data analysis and revised and edited the manuscript. All authors approved the final version of the manuscript. The corresponding author, $\mathrm{HMC}$, had access to all the data and had final responsibility for the decision to submit for publication.

Open Access This article is distributed under the terms of the Creative Commons Attribution Noncommercial License which permits any noncommercial use, distribution, and reproduction in any medium, provided the original author(s) and the source are credited.

\section{Appendix}

Inclusion and exclusion ICD-10 codes

$\begin{array}{ll}\begin{array}{ll}\text { Inclusion } \\ \text { S72.0 }\end{array} & \begin{array}{c}\text { Fracture neck of femur not otherwise } \\ \text { specified } \\ \text { Sertrochanteric fracture }\end{array} \\ \text { S72.2 } & \begin{array}{l}\text { Subtrochanteric fracture } \\ \text { Exclusion }\end{array} \\ \text { V01 to V99 } & \text { Transport accidents } \\ \text { W11 to W17 } & \begin{array}{l}\text { Falls from one level to another or } \\ \text { external collisions other than fall } \\ \text { from stairs or steps }\end{array} \\ \text { W20 to W49 } & \begin{array}{c}\text { Certain external causes of accidental } \\ \text { injury e.g. being struck by objects }\end{array} \\ \text { X70, X72 to X75, X79 } & \begin{array}{c}\text { Certain acts of intentional self harm } \\ \text { likely to cause fracture }\end{array} \\ \text { X91, X93 to X96, X99 } & \text { Assault } \\ \text { Y00-Y04, Y08, Y09,Y22 } & \text { Certain accidents of undetermined intent } \\ \text { to Y25, Y30 to Y32 } & \text { Injuries due to legal intervention and war } \\ \text { Y35 to Y36 } & \text { Sequelae of other accidents } \\ \text { Y86 } & \end{array}$




\section{References}

1. Lewis JD, Ferrara A, Peng T et al (2011) Risk of bladder cancer among diabetic patients treated with pioglitazone: interim report of a longitudinal cohort study. Diabetes Care 34:916-922

2. European Medicines Agency (2011) Questions and answers on the review of pioglitazone-containing medicines (Actos, Glustin, Competact, Glubrava and Tandemact). Available from www.ema. europa.eu/docs/en GB/document library/Medicine QA/2011/07/ WC500109179.pdf, accessed 12 December 2011

3. Neumann A, Weill A, Ricordeau P, Fagot JP, Alla F, Allemand H (2012) Pioglitazone and risk of bladder cancer among diabetic patients in France: a population-based cohort study. Diabetologia 55:1953-1962

4. Kahn SE, Zinman B, Lachin JM et al (2008) Rosiglitazoneassociated fractures in type 2 diabetes: an Analysis from a Diabetes Outcome Progression Trial (ADOPT). Diabetes Care 31:845-851

5. Home PD, Pocock SJ, Beck-Nielsen H et al (2009) Rosiglitazone evaluated for cardiovascular outcomes in oral agent combination therapy for type 2 diabetes (RECORD): a multicentre, randomised, open-label trial. Lancet 373:2125-2135

6. Nissen SE, Nicholls SJ, Wolski K et al (2008) Comparison of pioglitazone vs glimepiride on progression of coronary atherosclerosis in patients with type 2 diabetes: the PERISCOPE randomized controlled trial. JAMA 299:1561-1573

7. Dormandy J, Bhattacharya M, van Troostenburg de Bruyn AR (2009) Safety and tolerability of pioglitazone in high-risk patients with type 2 diabetes: an overview of data from PROactive. Drug Saf 32:187-202

8. Aubert RE, Herrera V, Chen W, Haffner SM, Pendergrass M (2010) Rosiglitazone and pioglitazone increase fracture risk in women and men with type 2 diabetes. Diabetes Obes Metab 12:716-721

9. Dormuth CR, Carney G, Carleton B, Bassett K, Wright JM (2009) Thiazolidinediones and fractures in men and women. Arch Intern Med 169:1395-1402

10. Habib ZA, Havstad SL, Wells K, Divine G, Pladevall M, Williams LK (2010) Thiazolidinedione use and the longitudinal risk of fractures in patients with type 2 diabetes mellitus. J Clin Endocrinol Metab 95:592-600

11. Solomon DH, Cadarette SM, Choudhry NK, Canning C, Levin R, Sturmer T (2009) A cohort study of thiazolidinediones and fractures in older adults with diabetes. J Clin Endocrinol Metab 94:2792-2798

12. Bilik D, McEwen LN, Brown MB et al (2010) Thiazolidinediones and fractures: evidence from translating research into action for diabetes. J Clin Endocrinol Metab 95:4560-4565

13. Loke YK, Singh S, Furberg CD (2009) Long-term use of thiazolidinediones and fractures in type 2 diabetes: a meta-analysis. CMAJ 180:32-39

14. Haentjens P, Magaziner J, Colón-Emeric CS et al (2010) Metaanalysis: excess mortality after hip fracture among older women and men. Ann Intern Med 152:380-390

15. Gronskag AB, Romundstad P, Forsmo S, Langhammer A, Schei B (2011) Excess mortality after hip fracture among elderly women in Norway: the HUNT study. Osteoporos Int 23:1807-1811
16. Scottish Diabetes Survey Monitoring Group (2009). Scottish diabetes survey 2009. Available from www.diabetesinscotland.org.uk/ Publications/Scottish\%20Diabetes\%20Survey\%202009.pdf, accessed on 16 November 2010

17. Kendrick S (1997) ISD The Scottish RECORD LINKAGE System. Available from www.isdscotland.org/isd/files/The $\% 20$ Scottish $\% 20$ Record\%20Linkage\%20System.doc, accessed 16 November 2011

18. Thompson WA Jr (1977) On the treatment of grouped observations in life studies. Biometrics 33:463-470

19. Rodriguez G (2012) Generalized linear models. Available from http://data.princeton.edu/wws509/notes/c7s6.html, accessed 11 May 2012

20. Hippisley-Cox J, Coupland C (2009) Predicting risk of osteoporotic fracture in men and women in England and Wales: prospective derivation and validation of QFracture scores. BMJ 339:b4229

21. Black DM, Kelly MP, Genant HK et al (2010) Bisphosphonates and fractures of the subtrochanteric or diaphyseal femur. N Engl $\mathrm{J}$ Med 362:1761-1771

22. Douglas IJ, Evans SJ, Pocock S, Smeeth L (2009) The risk of fractures associated with thiazolidinediones: a self-controlled caseseries study. PLoS Med 6:e1000154

23. Farrington CP, Anaya-Izquierdo K, Whitaker HJ, Hocine MN, Douglas I, Smeeth L (2011) Self-controlled case series analysis with event-dependent observation periods. J Am Stat Assoc 106:417-426

24. Hsiao FY, Mullins CD (2010) The association between thiazolidinediones and hospitalisation for fracture in type 2 diabetic patients: a Taiwanese population-based nested case-control study. Diabetologia 53:489-496

25. Bazelier MT, van Staa TP, Uitdehaag BM et al (2012) Risk of fractures in patients with multiple sclerosis: a population-based cohort study. Neurology 78:1967-1973

26. Tzoulaki I, Molokhia M, Curcin Vet al (2009) Risk of cardiovascular disease and all cause mortality among patients with type 2 diabetes prescribed oral antidiabetes drugs: retrospective cohort study using UK general practice research database. BMJ 339:b4731

27. Lecka-Czernik B (2010) Bone loss in diabetes: use of antidiabetic thiazolidinediones and secondary osteoporosis. Curr Osteoporos Rep 8:178-184

28. Robins JM, Hernan MA, Brumback B (2000) Marginal structural models and causal inference in epidemiology. Epidemiology $11: 550-560$

29. Food and Drug Administration (2011) FDA Drug Safety Communication: update to ongoing safety review of Actos (pioglitazone) and increased risk of bladder cancer. Available from www.fda.gov/ Drugs/DrugSafety/ucm259150.htm, accessed 12 December 2011

30. Cancer Research UK (2010) Bladder cancer statistics and outlook. Available from http://cancerhelp.cancerresearchuk.org/type/ bladder-cancer/treatment/bladder-cancer-statistics-and-outlook, accessed 12 December 2011

31. European Medicines Agency (2011) European Medicines Agency recommends new contraindications and warnings for pioglitazone to reduce small increased risk of bladder cancer. Available from www.ema.europa.eu/docs/en_GB/document_library/Press release/2011/07/WC500109176.pdf, accessed 15 December 2011 